\title{
Preliminary animal studies on observation of injured spinal cord with intraoperative ultrasound backscatter microscopy
}

\author{
NIU HaiJun, LI LiFeng, LIAO Kai, LI XiaoGuang ${ }^{*} \&$ FAN YuBo* \\ Key Laboratory of the Ministry of Education for Biomechanics and Mechanobiology, State Key Laboratory of Software Development Environment, \\ School of Biological Science and Medical Engineering, Beihang University, Beijing 100191, China
}

Received September 15, 2011; accepted January 10, 2012

\begin{abstract}
Many studies have shown that strategies of nerve regeneration and cell-based transplantation are valid based on animal models of spinal cord injury (SCI). To apply these strategies and bridge spinal cord defects, the identification and precise localization of lesions during spinal cord surgery is necessary. The aim of the present experiment was to evaluate the capabilities of ultrasound backscatter microscopy (UBM) in identifying morphologic changes after SCI. After laminectomy, high-resolution ultrasound images of the spinal cord were obtained in one normal and seven spinal cord-injured adult Wistar rats using a UBM system with a $55-\mathrm{MHz}$ center frequency scanner. Comparison between histoanatomic and UBM images was also performed. The results showed that UBM can identify cysts after the experimental SCI is removed in adult rats. In addition, the glial scar formed in secondary injury showed obvious hyperechoic speckle in the UBM image and correlated with the histoanatomic image. UBM has obvious clinical value in nerve regeneration and cell-based transplantation strategies in injured spinal cords.
\end{abstract}

ultrasound backscatter microscopy, spinal cord injury, cyst, glial scar

Citation: Niu H J, Li L F, Liao K, et al. Preliminary animal studies on observation of injured spinal cord with intraoperative ultrasound backscatter microscopy. Chin Sci Bull, 2012, 57: 2280-2284, doi: 10.1007/s11434-012-5219-7

Spinal cord injury (SCI) causes serious damage to the central nervous system. Although improvements in emergency and acute care with the progress of modern medicine have increased survival rates of SCI, the number of individuals who must cope with severe, lifelong disabilities has increased. These patients suffer greatly because of permanent sense and movement losses.

In the past decade, SCI research has resulted in many discoveries, especially nerve regeneration strategies and cell-based transplantation strategies, which have been validated in animal models of spinal cord injury [1-3], making the repair of injured spinal cords and functional recovery a potential goal. However, regeneration of spinal nerve fibers is difficult. Axonal recovery in the spinal cord is thwarted by two fundamental obstacles: inherently weak regenerative ability and a powerfully inhibitive post-injury environment of physical and chemical factors [4]. The most potent factor

*Corresponding authors (email: lixiaoguang@buaa.edu.cn; yubofan@buaa.edu.cn) is the glial scar and cyst produced with secondary SCI at the injury site. The glial scar is a collection of reactive cells (astrocytes, microglia, and meningeal fibroblasts, among others) and their expressed cell-surface and matrix molecules that surround the area of injury and ultimately hinder the advancement of all regenerating axons [5-7]. Some studies have confirmed that surgical glial scar resection for axonal regeneration is effective. However, to resect glial scars and bridge spinal cord defects, the precise localization and identification of glial scars during surgical manipulation is necessary $[8,9]$. Thus, how to obtain images with high quality and high resolution is vital for successful resection of glial scars and healing of spinal cord defects.

Magnetic resonance imaging (MRI) is the most widely employed diagnostic modality in neuroradiology. It can demonstrate the anatomical structure and associated problems in and around lesions during preoperative diagnoses. MRI facilitates the identification of correlations between histopathologic sequelae observed after SCI, such as edema, 
hemorrhage, and secondary degenerative changes including glial scar and cyst formation [10]. However, MRI provides only a reference because the spinal cord may undergo intraoperative deformation and excursion; thus, its intraoperative use is limited [5].

As a commonly used diagnostic tool, intraoperative ultrasound has been suggested for identification and characterization of spinal cord lesions and has been successfully used to guide intraspinal surgery for many years [11-15]. Compared with preoperative MRI, intraoperative ultrasound not only provides visualization of intraspinal abnormalities, but assesses the progress of spinal cord surgery before the operation is completed [16]. However, its limited image quality has limited its use. Thus, ultrasound backscatter microscopy (UBM) was developed. This technique allows for visualization of the internal structure of soft tissues at microscopic resolution [17-21]. Transducers with higher frequencies are used to obtain resolution 10 to 20 times greater than that of conventional ultrasonography, and structures as small as $20 \mu \mathrm{m}$ can be seen. This equipment is sensitive to subtle changes in tissues and is clinically used to diagnose small structural lesions, such as the internal structure of living tumor spheroids and the anterior structures of the intact eye.

The purpose of the present experiment was to explore (1) whether spinal cord lesions such as cysts and glial scars could be visualized more precisely with UBM, (2) whether the UBM image correlates the findings with those of light microscopy, and (3) the clinical value of intraoperative UBM in the guidance of spinal cord glial scar resection.

\section{Materials and experiment}

\subsection{Surgical procedure and animal care}

Eight adult Wistar rats (250-300 g) were used in these experiments. One normal rat was used to directly follow the ultrasound image acquisition process. The other seven rats were anesthetized by intraperitoneal injection of $6 \%$ chloral hydrate $(0.6 \mathrm{~mL} / \mathrm{kg}$ body weight $)$, and their skin was shaved before they were fixed in the prone position on the operating table. The spinous process T8 was identified as the center of the incision, and the skin and muscles were cut about $3 \mathrm{~cm}$ along the direction from head to tail. Under an operation microscope, the rats underwent sharp separation of the paravertebral muscles until their thoracic vertebrae from T7 to T9 were exposed; laminectomy was then performed at the exposed segment. The spinal dura mater was cut open, and the spinal cord was transected with a surgical blade at the T8 level, ensuring contact between the frontal and lateral walls of the vertebral canal. The muscles and skin were then sutured. Immediately following the operation, the rats were returned to their cages with easily accessible water and food at room temperature $\left(24-26^{\circ} \mathrm{C}\right)$ and were intraperitoneally injected with $1 \mathrm{~mL}$ of antibiotics once daily in the first postoperative week. The bladder was massaged twice daily after the surgery until normal bladder function was restored. This experimental procedure was approved by the Experimental Animal Center and Animal Care Committee, Beihang University, Beijing, China [22].

\subsection{Image acquisition}

Intraoperative UBM imaging was performed with a Vevo 770 (VisualSonics, Toronto, Canada) high-frequency UBM system. A 55-MHz center frequency transducer (RMV708) was employed. At a 4.5-mm focal distance, the axial resolution was $30 \mu \mathrm{m}$ with a $10.7-\mathrm{mm}$ depth of field. Two-dimensional images were acquired with an $8 \mathrm{~mm} \times 8 \mathrm{~mm}$ field of view at 46 frames per second.

All rats were used for UBM imaging after three months. The rats were anesthetized by intraperitoneal injection of $6 \%$ chloral hydrate $(0.6 \mathrm{~mL} / \mathrm{kg}$ body weight $)$. The skin and muscles were then incised approximately $3 \mathrm{~cm}$ around the original surgical lesion site. Laminectomy was performed to excise vertebrae $\mathrm{T} 7$ to $\mathrm{T} 9$ at the exposed segment. The bone window was opened approximately $5 \mathrm{~cm} \times 4 \mathrm{~cm}$ to expose the spinal cord.

Intraoperative UBM imaging was performed after the laminectomy, and the spinal cord was viewed (Figure 1). The surgical wound provided a basin that was filled with sterile gel. The ultrasound scan head was then inserted into the saline pool to provide images without actual tissue contact. Imaging was always performed in both the longitudinal and transverse planes [23,24].

\subsection{Histology}

Following ultrasound scanning, the aortic cannulae of two rats were immediately perfused with $150-200 \mathrm{~mL}$ of normal saline and $250-300 \mathrm{~mL}$ of $4 \%$ paraformaldehyde. The spinal cord was then carefully excised and fixed in the same fixative, $4 \%$ paraformaldehyde, for $8 \mathrm{~h}$ at $40^{\circ} \mathrm{C}$. The spinal tissue, including the lesional area, could be seen under a dissecting microscope. A spinal cord segment of approximately $4 \mathrm{~cm}$ was cut off using scissors. The lesion area in the center of the segment was ascertained, and the segment was embedded in epoxy resin. Epon-embedded sections $(8 \mu \mathrm{m})$ were cut horizontally, stained with NF and GFAP, and

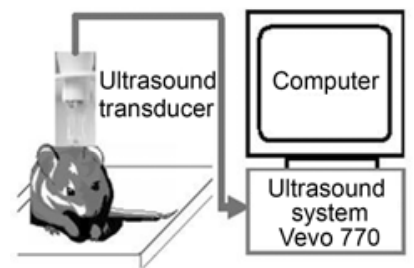

(a)

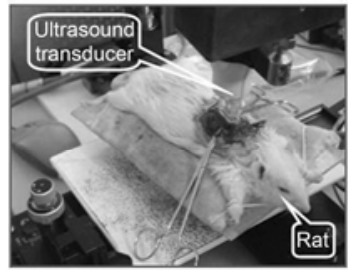

(b)
Figure 1 (a) A sketch of the UBM imaging system. (b) One Wistar rat is scanned using the UBM system. 
observed under a fluorescence microscope equipped with a digital camera system. In all experiments, image acquisition using UBM was successful, and there were no complications related to the use of the instrument.

\section{Results and discussion}

Figure 2(b) and (c) shows the longitudinal and transverse UBM images, respectively, of a normal rat thoracic spinal cord intraoperatively scanned through a gel-filled bone window. The image was compared with that obtained using a conventional B-mode sonographic probe $(10 \mathrm{MHz})$ (Figure 2(a)). It is obvious that the structures and contents of the spinal cord are precisely and sharply delineated in the UBM images. In Figure 2(b), the longitudinal scan primarily depicts the spinal cord white matter (hypoechoic region) with a small, longitudinally oriented hyperintense structure that reflects the gray matter of the lateral ventral horn. The ventral dura and vertebral bodies appear obvious in the hyperechoic region (white hollow arrow). The transverse scan in Figure 2(c) allows us to distinguish between the butterfly appearance of the spinal cord gray matter and the surrounding hypoechoic white matter. The dural surface around the spinal cord shows a hyperechoic region (white solid arrow).

Figure 3(a) shows a longitudinal section of thoracic spinal cord of one rat with acute injury. The spinal cord was transected with a surgical blade at the level of T8. The spinal cord wound is clear in the image. The tissue with the hypoechoic region is the spinal cord, which is surrounded by middle-level reflective blood and other fluid (BF). The ventral dura appears hyperechoic (white hollow arrow). Figure 3(a) also shows that the tissues were deformed and upheaved because the spinal cord and dura were transected.

Figure 3(b) shows a longitudinal section image of the thoracic spinal cord of one rat with chronic injury; the image was obtained using conventional B-mode ultrasonography. Figure 3(c) is a longitudinal section UBM image of the same rat. The resolution of the UBM image is better than that obtained by conventional B-mode sonography. The damaged location shows obvious hyperechogenicity and an irregular shape in Figure 3(c). No cyst cavity appeared during the secondary degenerative process in this rat. Figure 3(d) is a longitudinal section UBM image of the thoracic spinal cord of another rat with chronic injury. Many cysts developed during the secondary degenerative process. In addition to large cysts, all small cysts with diameters of 0.1$0.2 \mathrm{~mm}$ are clearly observed in the UBM image.

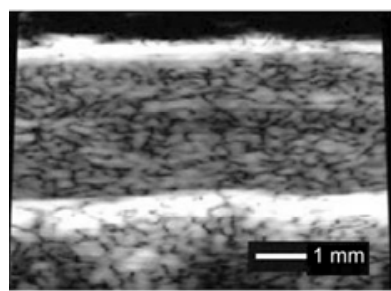

(a)

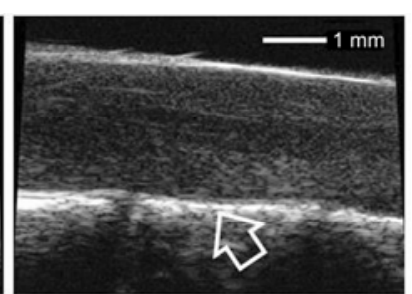

(b)

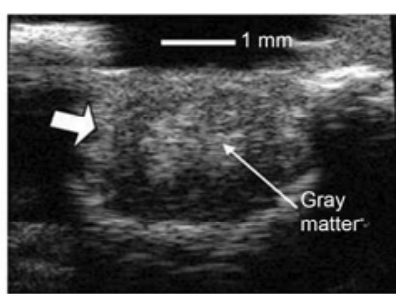

(c)

Figure 2 (a) A conventional B-mode ultrasound (10 MHz) image of a normal rat; (b) and (c) longitudinal and transverse section UBM images of the same normal spinal cord (white hollow arrow, vertebral bodies; white solid arrow, dural surface).
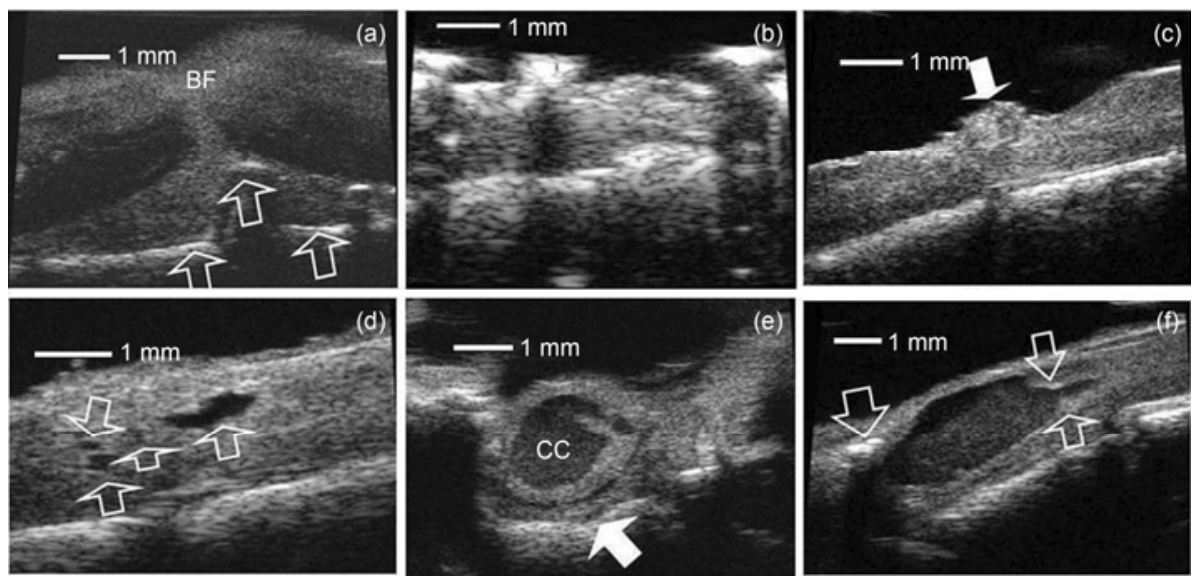

Figure 3 (a) Thoracic spinal cord longitudinal section UBM image of one rat with acute injury (white hollow arrow, ventral dural surface; BF, blood). (b) Longitudinal section conventional B-mode sonography and (c) UBM image of one rat with chronic injury (white solid arrow, chronic spinal cord injury). (d) Longitudinal transverse section UBM image of one rat with a chronically injured spinal cord (white hollow arrow, cyst cavity). (e) Transverse section UBM image and (f) longitudinal section UBM image of another rat with a chronically injured spinal cord (CC, cyst cavity; white solid arrow, ventral dural surface; white hollow arrow, glial scar). 
The spinal cord transverse and longitudinal section UBM images of one rat with serious chronic injury are shown in Figure 3(e) and (f), respectively. A large cyst can be seen in both the transverse and longitudinal images. The ventral dura appears hyperechoic (white solid arrow). Obvious hyperechoic speckles appear at the edge of the cyst cavity with homogeneous hypoechogenicity. These hyperechoic speckles differ from normal spinal cord tissue. These speckles were considered to be a glial scar formed by increased numbers of neuroglial cells.

To determine the imaging effect of glial scars in UBM images, histoanatomic and UBM images were compared. Figure 4(a) and (b) shows fluorescence microscopy photographs of spinal cord tissue segments, and Figure 4(c) shows the longitudinal section UBM image of a spinal cord with chronic injury. The photography reveals an obviously dense part of the neuroglial cell (white solid arrow). The dense neuroglial pad can also be seen in the UBM image. This finding could be helpful in resection of the glial scar following SCI.

\section{Conclusion}

Ultrasound imaging is an important tool that has been used to observe intraspinal abnormalities and assess the progress of spinal cord surgery before the operation is completed. Many publications have described the application of intraoperative ultrasound of the spinal cord $[12,15,16]$, but the image quality of conventional B-mode sonography has limited its use. Some researchers have suggested that ultra-highfrequency ultrasound should produce more detailed images of the spinal cord [25]. Based on this opinion, the present

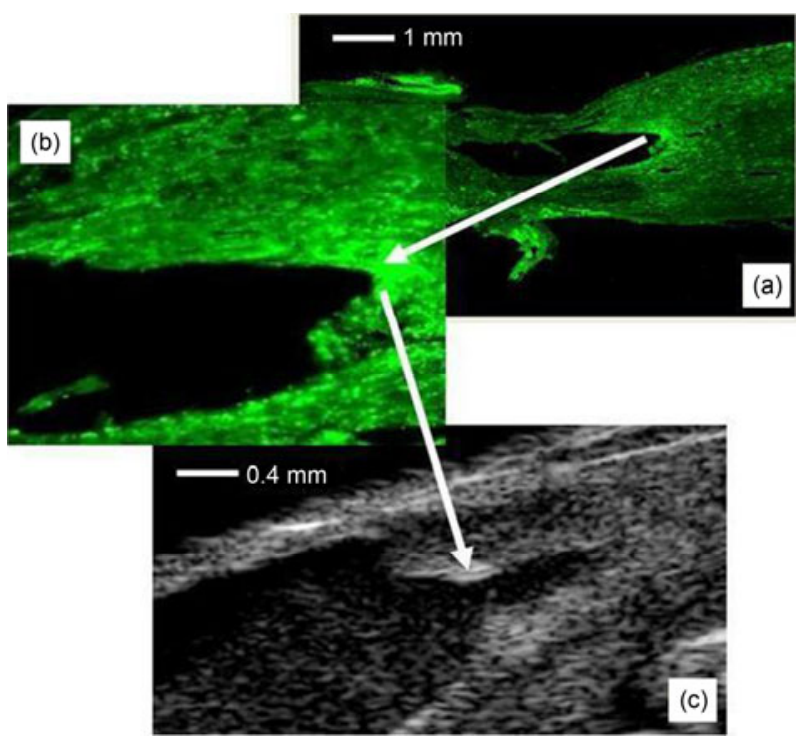

Figure 4 (a), (b) Fluorescence microscopy photograph of a spinal cord longitudinal section of one rat with chronic injury. (c) UBM image of a spinal cord longitudinal section of the same rat. experimental investigation was undertaken to evaluate the capabilities of UBM imaging to identify morphologic changes that occur after the experimental SCI is cut off in adult rats.

(1) Compared with common B-mode sonography, the quality and resolution of UBM images are obviously better. The structures and contents of the spinal cord of the rat model can be precisely and sharply delineated. (2) In the normal rat model, the spinal cord gray matter and surrounding white matter can possibly be distinguished in UBM images. In common B-mode sonography, this is impossible. (3) For acutely injured spinal cords, the spinal cord and surrounding blood can be obviously distinguished. (4) The most important finding comes from the observation of spinal cords with chronic injury. With the exception of small cysts, glial scars formed in secondary injury also showed obvious hyperechoic speckling in UBM images. Fluorescence microscopy photography of spinal cord tissue slices revealed that these hyperechoic speckles appearing in the UBM image represented the dense part of neuroglial cells. The UBM images correlated with the histoanatomic images.

However, higher frequency in ultrasound means both improved resolution and reduced tissue penetration. The diameter of the human spinal cord is obviously larger than that of rats. The feasibility of higher-frequency ultrasound in humans must be further explored. However, findings from this study provide useful information for further clinical experiments employing UBM imaging in nerve regeneration strategies and cell-based transplantation strategies in the injured spinal cord, which might become a relevant therapy for humans with SCI.

This work was supported by the National Natural Science Foundation of China (10772018) and the State Key Laboratory of Software Development Environment (SKLSDE-2011ZX-11).

1 Ramon-Cueto A, Cordero M I, Santos-Benito F F, et al. Functional recovery of paraplegic rats and motor axon regeneration in their spinal cords by olfactory ensheathing glia. Neuron, 2000, 25: 425-435

2 Teng Y D, Lavik E B. Functional recovery following traumatic spinal cord injury mediated by a unique polymer scaffold seeded with neural stem cells. Proc Natl Acad Sci USA, 2002, 99: 3024-3029

3 Li X G, Yang Z Y, Yang Y. Morphological and electrophysiological evidence for regeneration of transected spinal cord fibers and restoration of motor functions in adult rats. Chin Sci Bull, 2006, 51: 918-926

4 Fawcett J W. Overcoming inhibition in the damaged spinal cord. J Neurotrauma, 2006, 23: 371-383

5 Stichel C C, Hans W M. Experimental strategies to promote axonal regeneration after traumatic central nervous system injury. Prog Neurobiol, 1998, 56: 119-148

6 John W M, Cristina S. Spinal-cord injury. Lancet, 2002, 359: 417425

7 Rasouli A, Bhatia N, Dinh P, et al. Resection of glial scar following spinal cord injury. J Orthop Res, 2009, 27: 931-936

8 Fitch M T, Doller C, Combs C K. Cellular and molecular mechanisms of glial scarring and progressive cavitation: In vivo and in vitro analysis of inflammation-induced secondary injury after CNS trauma. J Neurol Sci, 1999, 19: 8182-8198

9 Wang S S, Hu Y Y, Luo Z J. Quantity analysis of local cavity and scar in rat spinal cord injury. Orthop J Chin, 2005, 6: 450-452 
10 Weber T, Vroemen M, Behr V, et al. In vivo high-resolution MR imaging of neuropathologic changes in the injured rat spinal cord. Am J Neuroradiol, 2006, 27: 598-604

11 Kane R A. Intraoperative ultrasonography history, current state of the art, and future directions. J Ultrasound Med, 2004, 23: 1407-1420

12 Sosna J, Barth M M, Kruskal J B, et al. Intraoperative sonography for neurosurgery. J Ultrasound Med, 2005, 24: 1671-1682

13 Lammertse D, Dungan D, Dreisbach J, et al. Neuroimaging in traumatic spinal cord injury: An evidence-based review for clinical practice and research. J Spinal Cord Med, 2007, 30: 205-214

14 Wang M Y, Levi A D, Green B A. Intradural spinal arachnoid cysts in adults. Surg Neurol, 2003, 60: 49-55

15 Zeidman S M, Tomlin J M, Rodriguez-Cruz L. Intraoperative spinal sonography techniques in neurosurgery. Tech Neurosurg, 2003, 8: 27-39

16 Kolstad F, Rygh O, MSelbekk T, et al. Three-dimensional ultrasonography navigation in spinal cord tumor surgery. J Neurosurg Spine, 2006, 5: 264-270

17 Foster F S, Pavlin C J, Lockwood G R, et al. Principles and applications of ultrasound backscatter microscopy. IEEE Trans Ultrason Ferroelec Freq Contr, 1993, 40: 608-617

18 Razuvaev A, Lund K, Roy J, et al. Noninvasive real-time imaging of intima thickness after rat carotid artery balloon injury using ultrasound biomicroscopy. Atherosclerosis, 2008, 199: 310-316

19 Sherar M D, Noss M B, Foster E S. Ultrasound backscatter microscopy images the internal structure of living tumour spheroids. Nature, 1987, 330: 493-495

20 Pavlin C J, Harasiewicz K, Sherar M D, et al. Clinical use of ultrasound biomicroscopy. Ophthalmology, 1991, 98: 287-295

21 Goessling W, North T E, Zon L I. Ultrasound biomicroscopy permits in vivo characterization of zebrafish liver tumors. Nat Methods, 2007, 4: $551-553$

22 Li X G, Yang Z Y, Yang Y. Studies on repairing of hemisected thoracic spinal cord of adult rats by using a chitosan tube filled with alginate fibers. Prog Nat Sci, 2006, 16: 1051-1055

23 Quencer R M, Morse B M, Green B A, et al. Intraoperative spinal sonography: Adjunct to metrizamide CT in the assessment and surgical decompression of posttraumatic spinal cord cysts. Am J Roentgenol, 1984, 142: 593-601

24 Knake J E, Gabrielsen T O, Chandler W F, et al. Real-time sonography during spinal surgery. Radiology, 1984, 151: 461-465

25 Gooding G A, Berger M S, Linkowski G D, et al. Transducer frequency considerations in intraoperative US of the spine. Radiology, 1986, 160: 272-273

Open Access This article is distributed under the terms of the Creative Commons Attribution License which permits any use, distribution, and reproduction in any medium, provided the original author(s) and source are credited. 